\title{
Deploying Machine Learning Techniques for Human Emotion Detection
}

\author{
Ali I. Siam $\left(\mathbb{D},{ }^{1}\right.$ Naglaa F. Soliman $\left(\mathbb{D},{ }^{2}\right.$ Abeer D. Algarni, ${ }^{2}$ Fathi E. Abd El-Samie, ${ }^{2}$ \\ and Ahmed Sedik (D) ${ }^{3}$ \\ ${ }^{1}$ Department of Embedded Network Systems Technology, Faculty of Artificial Intelligence, Kafrelsheikh University, \\ Kafr El-Sheikh, Egypt \\ ${ }^{2}$ Department of Information Technology, College of Computer and Information Sciences, \\ Princess Nourah Bint Abdulrahman University, Riyadh 84428, Saudi Arabia \\ ${ }^{3}$ Department of the Robotics and Intelligent Machines, Faculty of Artificial Intelligence, Kafrelsheikh University, \\ Kafr El-Sheikh, Egypt
}

Correspondence should be addressed to Naglaa F. Soliman; nfsoliman@pnu.edu.sa

Received 22 July 2021; Revised 12 October 2021; Accepted 22 October 2021; Published 2 February 2022

Academic Editor: Bai Yuan Ding

Copyright (c) 2022 Ali I. Siam et al. This is an open access article distributed under the Creative Commons Attribution License, which permits unrestricted use, distribution, and reproduction in any medium, provided the original work is properly cited.

Emotion recognition is one of the trending research fields. It is involved in several applications. Its most interesting applications include robotic vision and interactive robotic communication. Human emotions can be detected using both speech and visual modalities. Facial expressions can be considered as ideal means for detecting the persons' emotions. This paper presents a realtime approach for implementing emotion detection and deploying it in the robotic vision applications. The proposed approach consists of four phases: preprocessing, key point generation, key point selection and angular encoding, and classification. The main idea is to generate key points using MediaPipe face mesh algorithm, which is based on real-time deep learning. In addition, the generated key points are encoded using a sequence of carefully designed mesh generator and angular encoding modules. Furthermore, feature decomposition is performed using Principal Component Analysis (PCA). This phase is deployed to enhance the accuracy of emotion detection. Finally, the decomposed features are enrolled into a Machine Learning (ML) technique that depends on a Support Vector Machine (SVM), k-Nearest Neighbor (KNN), Naïve Bayes (NB), Logistic Regression (LR), or Random Forest (RF) classifier. Moreover, we deploy a Multilayer Perceptron (MLP) as an efficient deep neural network technique. The presented techniques are evaluated on different datasets with different evaluation metrics. The simulation results reveal that they achieve a superior performance with a human emotion detection accuracy of $97 \%$, which ensures superiority among the efforts in this field.

\section{Introduction}

Recognition of human emotions is a vital phase, which is involved in several applications such as augmented and virtual reality $[1,2]$, advanced driver assistance systems [3], human computer interaction [4], and security systems [5-7]. Humans have several ways of interpreting the emotions of others, such as speech and linguistic aspects [8] and facial expressions [9-11]. Furthermore, emotions can be detected based on gaze direction [12] and biosignals including electroencephalogram (EEG) and electrocardiogram (ECG). Emotional expressions are used for intelligent HumanRobot Interaction (HRI). Emotion analysis can also be used to track the students' emotions to enhance the learning environment. Therefore, the students can learn better using this approach. Such information obtained through emotion analysis is useful in monitoring of the overall mood of a group of persons to identify any destructive events [13]. In human interaction, $7 \%$ of the affective information is conveyed by words, $38 \%$ is conveyed by speech tone, and 
$55 \%$ is conveyed by facial expressions [14]. Therefore, the facial emotion analysis can be a dependable approach to recognize human emotions for HRI applications.

The robot vision issue can be handled using thermal images [15-17] and RGB images [18]. This paper presents a real-time study for emotion detection and deployment in robotic vision applications. The proposed approach consists of four phases: preprocessing, feature extraction and selection, feature decomposition, and classification. Feature extraction and selection is carried out by MediaPipe face mesh algorithm. This algorithm is based on real-time deep learning. In addition, the feature decomposition phase is performed by PCA. This phase is deployed to enhance the accuracy of emotion detection. It is required to decompose the extracted features using the Singular Value Decomposition (SVD). Finally, the obtained features are enrolled into a selected classifier. In addition, an MLP deep neural network is utilized. The introduced techniques are assessed on different datasets with the help of different evaluation metrics. Moreover, this paper introduces a hardware implementation of the proposed models. The main contributions of this work can be summarized as follows:

(1) A novel fast and robust emotion detection framework for robotic vision applications is proposed.

(2) Emotion face mesh is introduced depending on automatic key point determination from face images.

(3) Key point angular encoding is presented to generate sensitive and distinguishable angular features.

(4) Emotion classification is performed depending on various machine learning techniques.

(5) A brief comparison is made between the deployed techniques in terms of accuracy, scalability, and processing time.

The remaining parts of this paper are organized as follows. Section 2 covers the works introduced in the literature. Section 3 shows the datasets utilized in this work. Furthermore, the proposed methodology is discussed in Section 4, and its simulation results are given in Section 5. Moreover, the result discussion highlights the performance of the proposed approach among the works in the literature in Section 6. Finally, the paper concluding remarks are given in Section 6.

\section{Related Work}

Several researchers presented their frameworks to handle the issue of HRI. The work in [19] offers a conditional-generative-adversarial-network-based (CGAN-based) framework to reduce intraclass variances by managing facial expressions individually, while simultaneously learning generative and discriminative representations. A generator $G$ and three discriminators make up this architecture (Di, Da, and Dexp). Any query face image is transformed into a prototypic facial expression form with certain factors kept by the generator $G$. An accuracy of $81.83 \%$ was achieved. A model based on $\mathrm{CNN}$ was proposed in the work of [20]. It was designed for smile detection, emotion recognition, and gender classification. Therefore, it is considered as a multi-task model. It achieved an accuracy of $71.03 \%$.

Some efforts have been presented for emotion detection using deep learning. The work in [21] introduced a deep CNN to deploy a facial expression recognition system. This system can automatically extract the features of facial expressions to allow automatic recognition. In addition, it consists of input, preprocessing, recognition, and output modules. Furthermore, it was used to simulate and assess the recognition performance under the effect of several aspects such as network structure, learning rate, and preprocessing on both the Japanese Female Facial Expression (JAFFE) dataset and the Extended Cohn-Kanade $(\mathrm{CK}+)$ dataset. To make the results more convincing, the authors used the k-Nearest Neighbor $(\mathrm{KNN})$ technique. For JAFFE and CK+ datasets, the performance accuracies are $76.7442 \%$ and $80.303 \%$, respectively. Another model was proposed in [22]. It was tested on a facial expression dataset of HDR images, considering a collection of faces under different lighting conditions. It is based on SVM, Local Binary Patterns (LBPs), and appearance. It works depending on the Speeded-Up Robust Feature (SURF) transform to conduct the emotion recognition task. This model revealed accuracy levels up to $80 \%$. In [23], the authors presented a model for submission to the fifth Emotion Recognition in the Wild (EmotiW 2017) group-level emotion recognition subchallenge. They deployed a CNN to extract features from the detected face images. Another role for the $\mathrm{CNN}$ is to be trained for the face identification task, rather than traditional pretraining on emotion recognition problems. In the final pipeline, an ensemble of Random Forest (RF) classifiers was learned to predict an emotion score using an available training set. This model achieved an accuracy of $75.4 \%$ on the validation data.

Another trend in this field is to detect emotions from videos. The authors of [24] presented a hybrid deep learning model for emotion detection from videos. A spatial CNN is used for processing of static facial images and a temporal CNN for optical flow images. These two processing branches are used to learn high-level spatial and temporal features on video segments, separately. These two CNNs are fine-tuned using pretrained $\mathrm{CNN}$ models and target video facial expression datasets. A deep fusion network, which is deployed using a Deep Belief Network (DBN) model, fuses the collected features from the segment-level spatial and temporal branches. The obtained fused features are enrolled into a linear SVM for facial expression classification tasks. The authors achieved an accuracy of $75.39 \%$. Moreover, another video-based emotion detection algorithm was presented in [25]. The authors investigated different ways for pooling spatial and temporal data. For video-based face expression identification, they discovered that pooling spatial and temporal information together is more efficient. Unlike the framework given in [24], this work is end-to-end trainable for whole-video recognition. The goal of this framework is to create a trainable deep neural network framework for pattern identification that integrates spatial and temporal information from video using CNNs and LSTMs. This framework achieved an accuracy of $65.72 \%$. 


\section{Dataset Description}

The proposed models are evaluated on three datasets: Cohn-Kanade $(\mathrm{CK}+)$ [26], Japanese Female Facial Expression (JAFFE) [27], and Real-world Affective Faces Database (RAF-DB) [28]. A description of each of them is given below.

3.1. Cohn-Kanade $[\mathrm{CK}+]$. The $\mathrm{CK}+$ dataset [26] consists of 593 video sequences from 123 participants. Each sequence contains images beginning from onset (neutral frame) and progressing to the peak expression (last frame). The label associated with each sequence is depicted from the peak expression. The dataset contains images for seven different expressions: anger, contempt, fear, disgust, happiness, surprise, and sadness. The images have a resolution of $640 \times 480$ pixels. In this work, the images are cropped into $48 \times 48$ pixels to focus on the subject face. Figure 1 shows sample images for each expression.

3.2. Japanese Female Facial Expression (JAFFE). The JAFFE dataset [27] has 213 photos of ten different female actors posing for seven different facial expressions. There are six primary expressions: happiness, sadness, surprise, anger, disgust, and fear, plus one neutral expression. The images have a resolution of $256 \times 256$ pixels. Figure 2 shows sample images for each expression.

3.3. Real-World Affective Face Database (RAF-DB). RAF-DB [28] contains 15,339 facial images with uncontrolled poses and illumination from thousands of individuals of different ages and races. The images within the RAF-DB are labeled by approximately 40 annotators. The database includes six basic expressions plus a neutral expression. Sample images from RAF-DB are shown in Figure 3.

\section{Proposed Methodology}

This paper presents an emotion detection approach based on deep and machine learning techniques. The main idea of this approach is to deploy deep learning as an automatic key point generator using MediaPipe technique. Hence, a sensitive mathematical process is performed to encode the generated key points into a set of distinguishable features. In addition, different machine learning techniques are implemented on the extracted features to perform the classification task. The proposed approach consists of four main phases. The first phase is image preprocessing in which a super-resolution task is carried out using SRGAN. In the second phase, we deploy MediaPipe to generate key landmarks on the face images. Furthermore, we present a key landmark analysis and an angular encoding module. This module contains three subphases (key landmark selection, emotional mesh generation, and mesh angular encoding). The main idea of this module is to generate an emotional mesh that connects the selected key landmarks. Hence, the obtained mesh is encoded into angular values to generate a feature map. Moreover, the generated feature map is enrolled into a classifier to be discriminated into six categories. Figure 4 represents the proposed framework.

4.1. Preprocessing. Generally, the images that are captured by robotic vision devices have a limited resolution due to the hardware limitations of cameras involved in such systems. Furthermore, most of the available datasets for human emotion recognition are down-sized because of the storage limitations. Therefore, the first module in the proposed approach is the super-resolution. In addition, the proposed approach involves angular feature extraction from the geometry of the face images, which requires a clarified representation of the landmarks and boundaries of the face images to allow proper facial emotion recognition. SRGAN [29], a Generative Adversarial Network (GAN) for image Super-Resolution (SR), is employed in the current research to increase the perceptual quality of images prior to further processes. With SRGAN, the images are super-resolved with a $4 x$ upscaling factor, while minimizing the Mean SquareError (MSE) between the super-resolved and original images and maximizing the Peak Signal-to-Noise Ratio (PSNR).

Figure 5 illustrates the preprocessing step by employing the SRGAN. The figure displays an original image selected from the $\mathrm{CK}+$ dataset and the corresponding super-resolved image after SRGAN. The original image size is $48 \times 48$ pixels, and the super-resolved image size is $192 \times 192$ pixels.

4.2. Key Landmark Generation. The process of key landmark generation is performed using deep MediaPipe technique. MediaPipe [30] is an open-source ML framework developed by Google and devoted to building real-life computer vision applications. MediaPipe capabilities allow developers to focus on algorithm or model development, while using MediaPipe to iteratively improve their application with results that are consistent across different devices and platforms [31]. Solutions that are currently implemented with MediaPipe include face detection, face mesh annotation, iris localization, hand detection, pose estimation, hair segmentation, object detection and tracking, and 3D object detection (Objectron). These solutions are released in different platforms: mobile (Android and iOS), $\mathrm{C}++$, Python, and JS. Real-life examples of ML solutions in MediaPipe are shown in Figure 6.

In the current work, the face mesh solution from the MediaPipe framework is employed to annotate the landmarks and boundaries of the face. Face mesh calculates 468 3D face landmarks in real time. It uses $\mathrm{ML}$ to infer 3D surface geometry using just a single camera input without a specialized depth sensor [32]. The solution provides a realtime performance, even on mobile devices. Figure 7 displays an image selected from the JAFFE dataset with the 468 facial landmarks annotated on the image.

4.3. Proposed Key Landmark Analysis and Angular Encoding. This paper presents a key landmark analysis and an angular encoding module. This module contains three subphases 

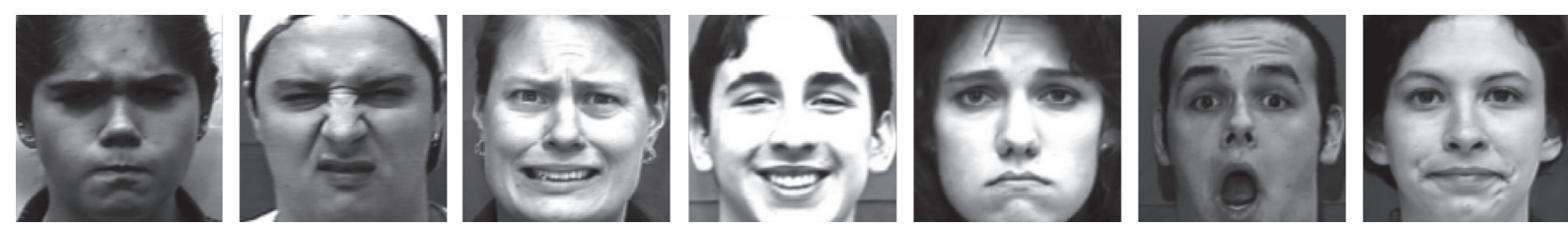

Figure 1: Examples of images from $\mathrm{CK}+$ dataset.

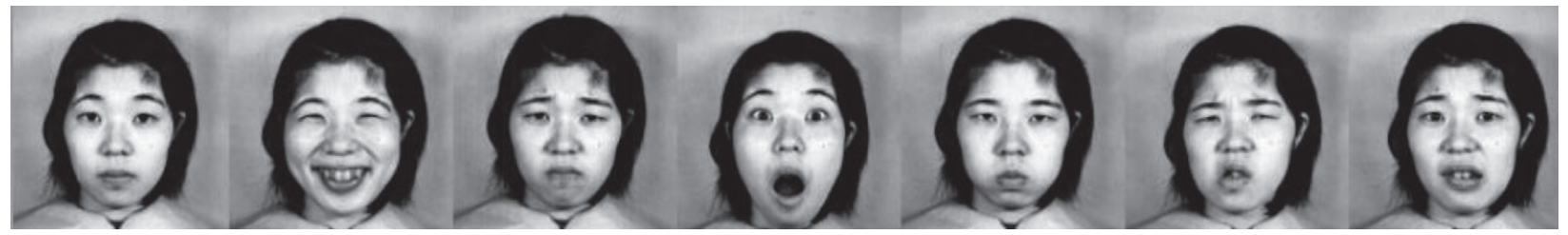

Figure 2: Examples of images from JAFFE dataset.
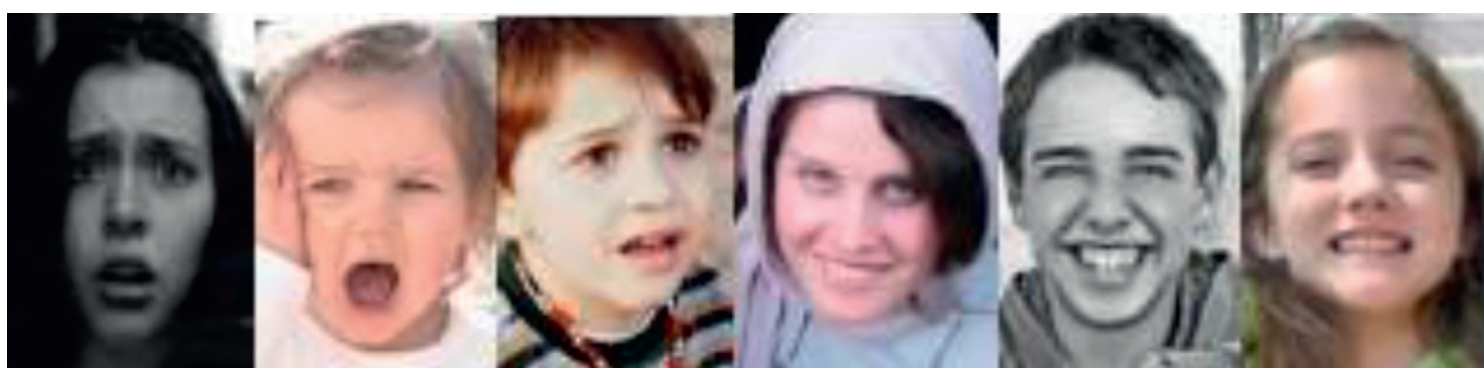

Figure 3: Examples of images from RAF-DB.

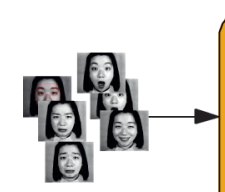

Input Images

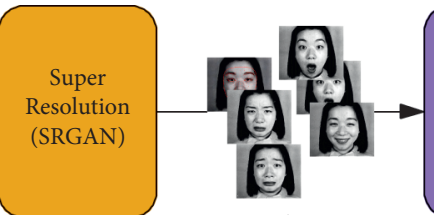

Resized Images
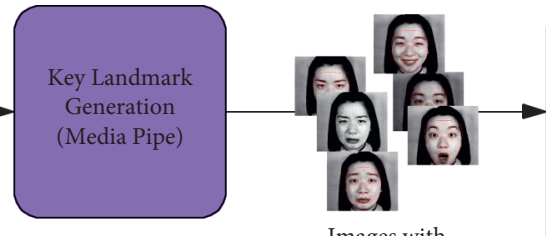

Images with
Classification

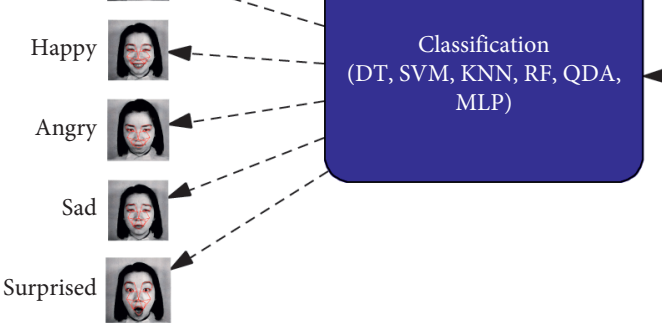

FIgURE 4: The proposed framework.

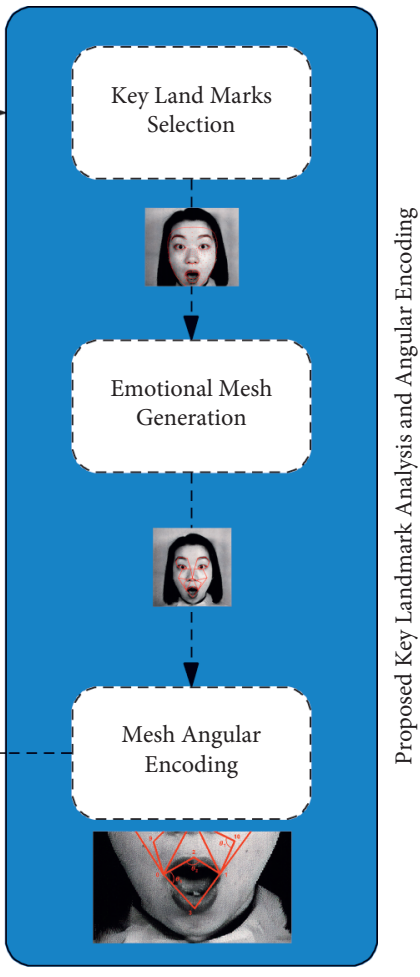

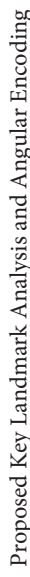



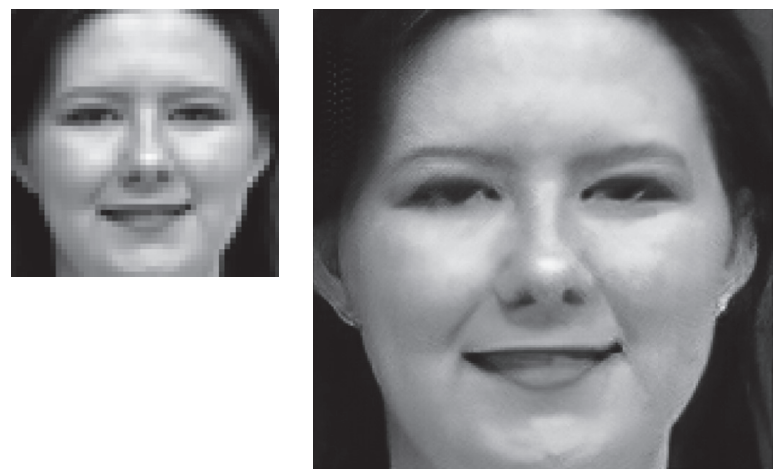

(a)

(b)

FIGURE 5: Preprocessing step (super-resolution): (a) original image [48 $\times 48]$; (b) $4 x$ upscaled super-resolved image using SRGAN $[192 \times 192]$.
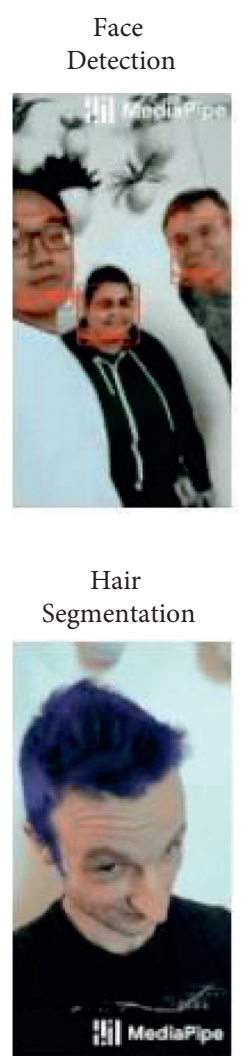

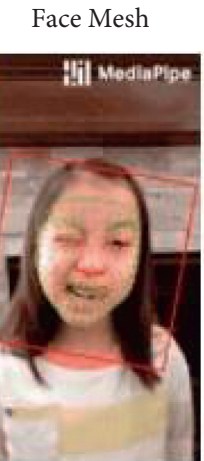

Object

Detection

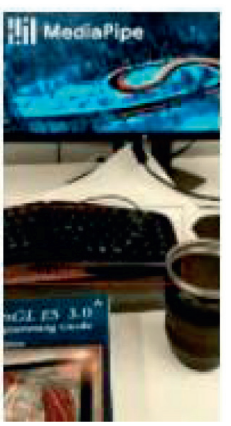

Iris

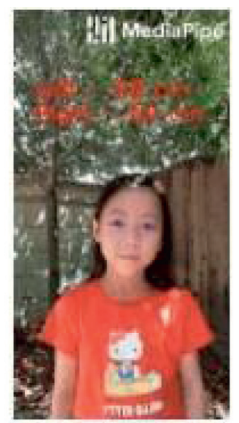

Box Tracking

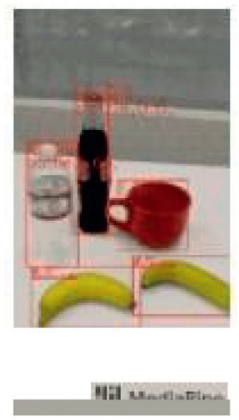

Hands

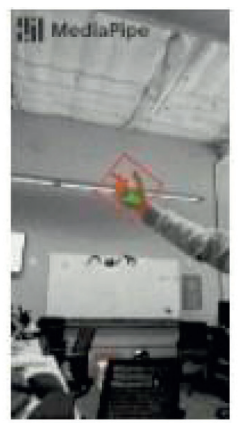

Instant Motion Tracking

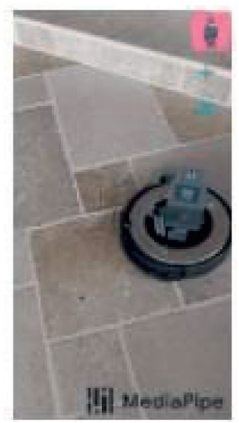

Pose

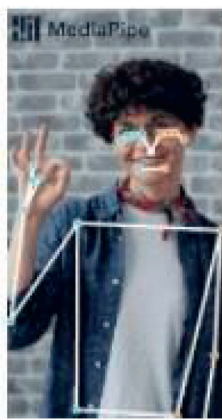

Objectron

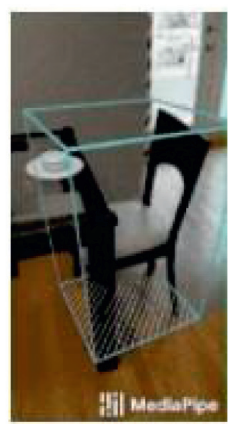

Holistic

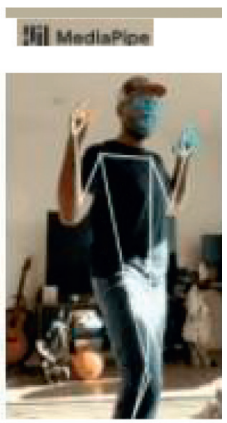

KNIFT

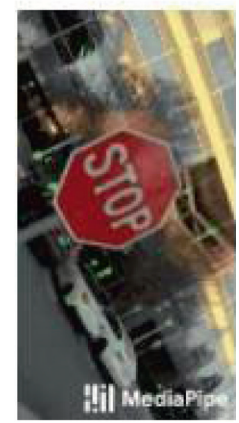

Figure 6: Different examples of ML solutions in MediaPipe [30].

(key landmark selection, emotional face mesh generation, and mesh angular encoding). The main idea of this module is to generate an emotional mesh, which connects the selected key landmarks. Hence, the obtained mesh is encoded into angular values to generate a feature map. In the following subsections, a discussion for each step in this module is presented.

4.3.1. Key Landmark Selection. As discussed earlier, the MediaPipe face mesh solution provides face detection capability and 468 facial landmarks spread over the face, along with their locations ( $x$ and $y$ coordinates for each detected landmark). In the proposed model, only 27 key landmarks are selected from the 468 detected landmarks. These key landmarks are used later to define the vertices of the emotion face mesh. Table 1 describes the selected key landmarks and the corresponding MediaPipe landmark IDs. The 27 key landmarks and their locations on a test face image are shown in Figure 8.

The selection of the key landmarks and their locations is based on the Facial Action Coding System (FACS) [33, 34], which encodes movements of individual facial muscles. It can be used to describe facial actions that make up an 


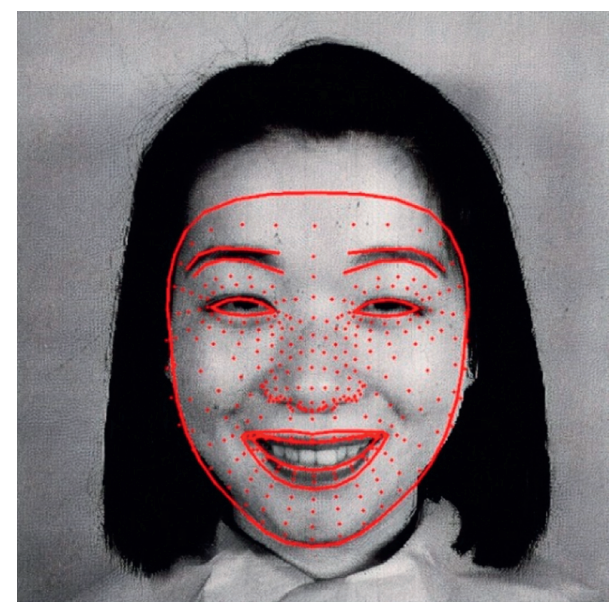

Figure 7: An image with 468 annotated landmarks using MediaPipe face mesh.

TABLE 1: Selected key landmarks (vertices) and the corresponding MediaPipe landmarks.

\begin{tabular}{lcc}
\hline Key landmark & MediaPipe landmark & Description \\
ID & ID & Mouth end (right) \\
\hline 0 & 61 & Mouth end (left) \\
1 & 292 & Upper lip (middle) \\
2 & 0 & Lower lip (middle) \\
3 & 17 & Right cheek \\
4 & 50 & Left cheek \\
5 & 280 & Nose right end \\
6 & 48 & Nose tip \\
7 & 4 & Nose left end \\
8 & 289 & Upper jaw (right) \\
9 & 206 & Upper jaw (left) \\
10 & 426 & Right eye (inner) \\
11 & 133 & Right eye (outer) \\
12 & 130 & Right upper eyelid \\
13 & 159 & (middle) \\
& & Right lower eyelid \\
14 & 145 & (middle) \\
15 & 362 & Left eye (inner) \\
16 & 359 & Left eye (outer) \\
17 & 386 & Left upper eyelid \\
& & (middle) \\
18 & 374 & Left lower eyelid \\
19 & 334 & (middle) \\
20 & 122 & Nose bridge (right) \\
21 & 351 & Nose bridge (left) \\
22 & 105 & Right eyebrow (outer) \\
23 & & Right eyebrow (middle) \\
25 & Right eyebrow (inner) \\
26 & Left eyebrow (outer) \\
\hline & & Left eyebrow (middle) \\
& Left eyebrow (inner) \\
\hline & &
\end{tabular}

expression based on changes in facial muscles regardless of emotion. The movement of particular facial muscles, known as Action Units (AUs), is encoded by FACS. This requires unique instantaneous changes in facial appearance [35]. Table 2 describes the facial emotion-related

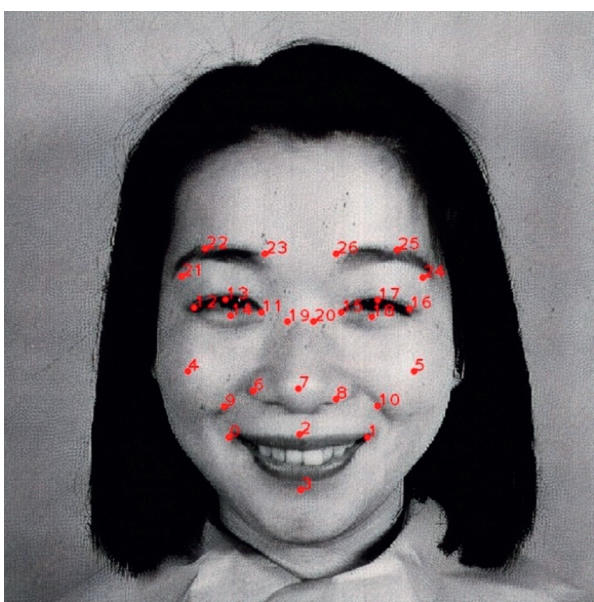

Figure 8: The 27 key landmarks and their locations.

TABLE 2: List of AU codes and FACS descriptors.

\begin{tabular}{lc}
\hline AU & FACS name \\
\hline 1 & Inner brow raiser \\
2 & Outer brow raiser \\
4 & Brow lowerer \\
5 & Upper lid raiser \\
6 & Cheek raiser \\
7 & Lid tightener \\
8 & Lips toward each other \\
9 & Nose wrinkler \\
10 & Upper lip raiser \\
11 & Nasolabial deepener \\
12 & Lip corner puller \\
13 & Sharp lip puller \\
14 & Dimpler \\
15 & Lip corner depressor \\
16 & Lower lip depressor \\
17 & Chin raiser \\
18 & Lip pucker \\
19 & Tongue show \\
20 & Lip stretcher \\
21 & Neck tightener \\
22 & Lip funneler \\
23 & Lip tightener \\
24 & Lip pressor \\
25 & Lips part \\
26 & Jaw drop \\
27 & Mouth stretch \\
28 & Lip suck \\
29 & Jaw thrust \\
30 & Jaw sideways \\
31 & Jaw clencher \\
\hline & \\
\hline &
\end{tabular}

AUs and the corresponding FACS names. A graphicbased demonstration for FACS with isolated AUs is illustrated in [36]. Hence, facial emotions can be represented using reliable combinations of different AUs, as demonstrated in Table 3. Each key landmark location is chosen such that it is more probably affected by a specific emotion-related AU, which seeks better recognition of facial expressions. 
Table 3: Emotion description in terms of AUs.

\begin{tabular}{lc}
\hline Emotion & AU \\
\hline Happiness & $6+12$ \\
Sadness & $1+4+15$ \\
Surprise & $1+2+5+26$ \\
Fear & $1+2+4+5+7+20+26$ \\
Anger & $4+5+7+23$ \\
Disgust & $9+15+17$ \\
Contempt & $12+14$ \\
\hline
\end{tabular}

4.3.2. Emotional Mesh Generation. After selection of the key landmarks, emotion face mesh is created, consisting of 27 vertices inferred from the selected key landmarks. Edges of the emotion face mesh, which define the connections between vertices, are drawn to establish a closed mesh structure. Table 4 defines the edges that constitute the emotion face mesh, as well as the start and end vertices for each edge. The vertices IDs are defined in Table 1. The mesh yields 27 vertices and 38 edges. Deformation of emotion face mesh measured by the deviation of angles between edges reflects facial muscle contraction and relaxation, which will be used to identify facial emotions. Figure 9 displays the emotion face mesh for sample images selected from the JAFFE dataset with different emotions.

4.3.3. Mesh Angular Encoding. After acquiring the key landmarks and establishing the emotion face mesh, we use the mesh to extract the relevant features for emotion classification. The relevant features employed are geometric features, since most emotions can be detected from geometric changes. Ten features are extracted, defining angles between specific edges of the emotion face mesh. The angles are represented in degrees in the range of $\left(0^{\circ}, 360^{\circ}\right)$. These features are then fed to the ML classifiers to learn from them to identify each emotion. The low dimensionality of features (10 features) makes them more resistant to local facial changes. In addition, the classifiers can be trained in a much shorter time. Moreover, the overall complexity of the proposed framework is significantly reduced. The list of angles taken as discriminant features for emotion classification, and the three vertices IDs forming each angle are given in Table 5. An example depicting the angular features and their locations on a test face image is shown in Figure 10.

The angle between the three vertices can be computed as follows (consider Figure 11).

The angle $\theta$ between the line (edge) connecting $P_{2}$ and $P_{3}$ and the line (edge) connecting the points $P_{2}$ and $P_{1}$ is unknown.

The angle $\beta$ between the line $P_{2}-P_{3}$ and the $X$-axis can be computed as

$$
\beta=\tan ^{-1}\left(\frac{y_{3}-y_{2}}{x_{3}-x_{2}}\right) .
$$

Similarly, the angle $\alpha$ between the line $P_{2}-P_{1}$ and the $X$-axis can be computed as

$$
\alpha=\tan ^{-1}\left(\frac{y_{1}-y_{2}}{x_{1}-x_{2}}\right) .
$$

TABLe 4: Edges and vertices of the emotion face mesh.

\begin{tabular}{lc}
\hline Edge & Connected vertices IDs \\
\hline 1 & $(0,2)$ \\
2 & $(0,3)$ \\
3 & $(1,2)$ \\
4 & $(1,3)$ \\
5 & $(7,6)$ \\
6 & $(7,8)$ \\
7 & $(6,4)$ \\
8 & $(8,5)$ \\
9 & $(6,9)$ \\
10 & $(9,0)$ \\
11 & $(4,0)$ \\
12 & $(8,10)$ \\
13 & $(10,1)$ \\
15 & $(7,19)$ \\
16 & $(7,20)$ \\
17 & $(7,0)$ \\
18 & $(7,1)$ \\
19 & $(19,23)$ \\
20 & $(19,14)$ \\
21 & $(23,22)$ \\
22 & $(22,21)$ \\
23 & $(21,12)$ \\
24 & $(12,13)$ \\
25 & $(12,14)$ \\
26 & $(11,13)$ \\
27 & $(11,14)$ \\
28 & $(14,4)$ \\
29 & $(20,26)$ \\
30 & $(26,25)$ \\
31 & $(25,24)$ \\
32 & $(24,16)$ \\
33 & $(16,17)$ \\
35 & $(16,18)$ \\
36 & $(15,17)$ \\
37 & $(15,18)$ \\
& $(18,20)$ \\
38 & $(18,5)$ \\
&
\end{tabular}

Hence, the angle $\theta$ will be

$$
\theta=\beta-\alpha=\tan ^{-1}\left(\frac{y_{3}-y_{2}}{x_{3}-x_{2}}\right)-\tan ^{-1}\left(\frac{y_{1}-y_{2}}{x_{1}-x_{2}}\right) .
$$

Using the above procedure, ten angles between prescribed edges in the emotion face mesh are computed, and then used for classification. Angle values are all positive, where negative values can be avoided by adding $360^{\circ}$ to the values. Furthermore, the generated feature maps are redistributed using PCA to enhance their distribution.

4.4. Classification. In this work, we develop an automated facial expression identifier to recognize human emotions for robotic vision applications. Discriminant features extracted from a face (Section 4.3) are fed to classifiers to recognize the emotion in the given face. DT, KNN, a multiclass SVM [37], Gaussian NB, MLP with backpropagation, QDA, RF, and LR classifiers are used for classification. The trial-and-error method and grid-search [38] are conducted to identify the 

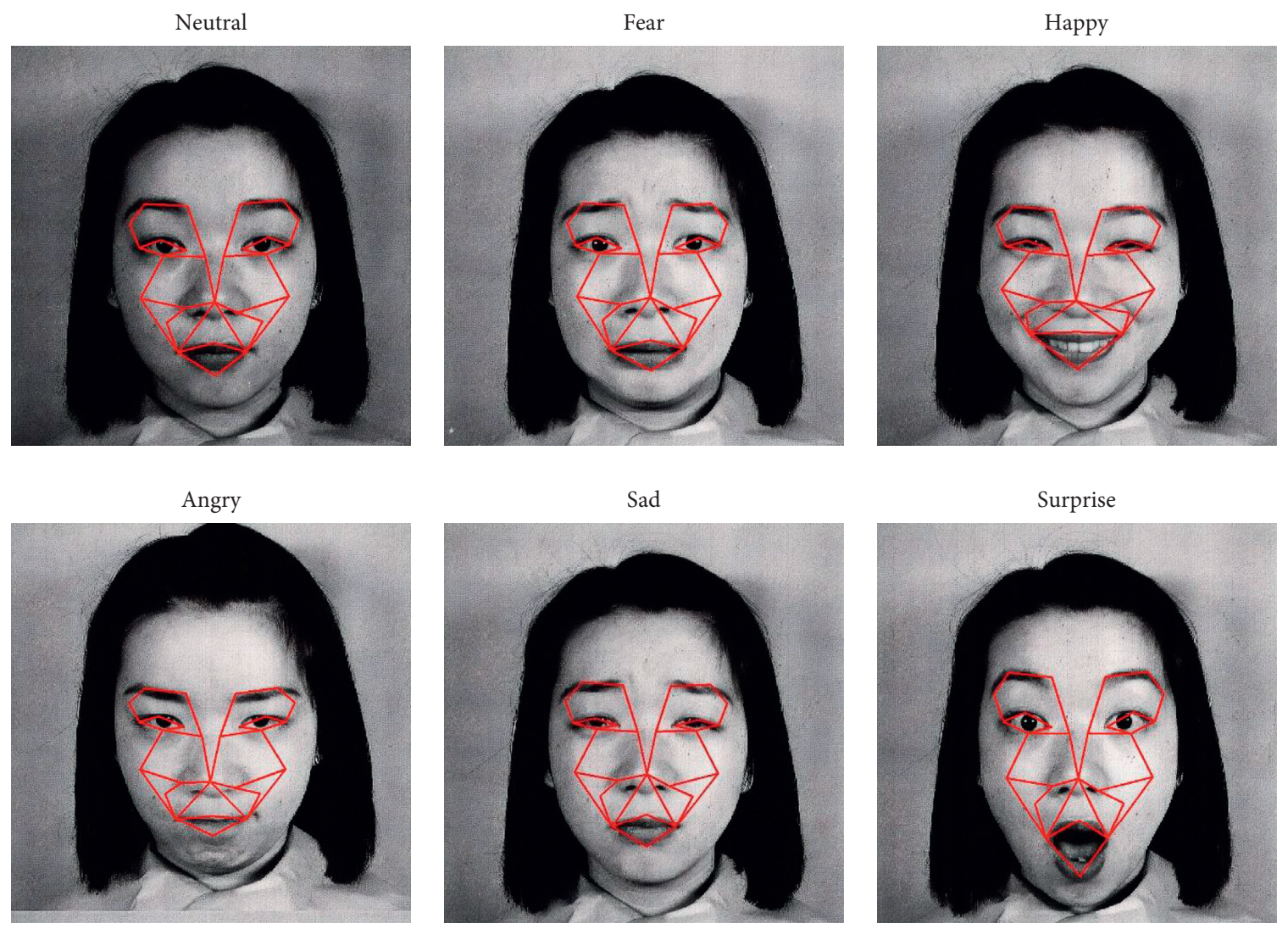

FiguRE 9: Samples of emotion face mesh for different emotions.

TABLE 5: List of features (angles) and the three enclosing vertices.

\begin{tabular}{lc}
\hline Features & Enclosing vertices IDs \\
\hline$\theta_{1}$ & $(2,0,3)$ \\
$\theta_{2}$ & $(0,2,1)$ \\
$\theta_{3}$ & $(6,7,8)$ \\
$\theta_{4}$ & $(9,7,10)$ \\
$\theta_{5}$ & $(0,7,1)$ \\
$\theta_{6}$ & $(1,5,8)$ \\
$\theta_{7}$ & $(1,10,8)$ \\
$\theta_{8}$ & $(13,12,14)$ \\
$\theta_{9}$ & $(21,22,23)$ \\
$\theta_{10}$ & $(6,19,23)$ \\
\hline
\end{tabular}

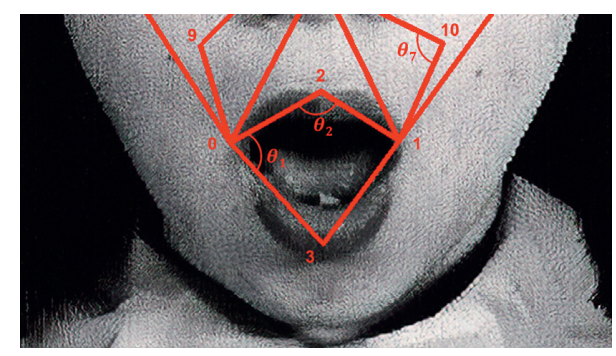

Figure 10: An example showing the features $\left(\theta_{1}, \theta_{2}\right.$, and $\left.\theta_{7}\right)$ and their locations.

optimal structure and hyperparameters of classifiers. In addition, 10 -fold cross-validation is employed to estimate the optimal hyperparameter combinations to avoid

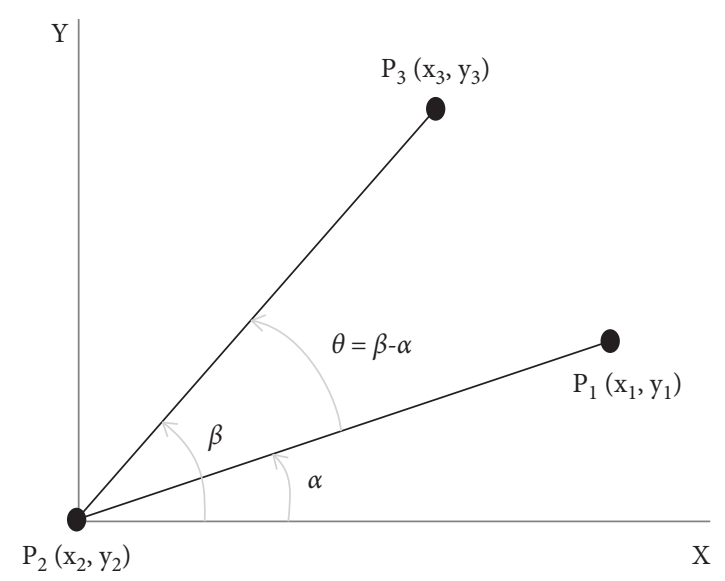

Figure 11: Computing the angle between two lines (edges).

overfitting. The optimal hyperparameters of classifiers adopted in the current work are investigated in Table 6.

The images in the dataset are divided into two parts: training part and testing part. The training part is used to train/validate the classifier, and the testing part is used to test the performance of the classifier. The splitting scheme is 80 / 20 , as shown in Figure 12. The 10-fold cross-validation adopted in the current model employs further splitting of the training part into ten folds (subsets). After that, nine folds are used to train the classifier, while the remaining fold is used to validate the training. This process continues until 
TABLE 6: List of hyperparameters of adopted classifiers.

\begin{tabular}{|c|c|}
\hline Classifier & Hyperparameters \\
\hline DT & $\begin{array}{c}\text { Criterion: "gini" } \\
\text { min_samples_leaf: } 1 \\
\text { min_samples_split: } 2 \\
\text { ccp_alpha: } 0\end{array}$ \\
\hline KNN & $\begin{array}{c}\text { n_neighbors: } 1 \\
\text { leaf_size: } 30 \\
\text { Metric: “minkowski” } \\
\text { p: } 2 \\
\text { Weights: “uniform" }\end{array}$ \\
\hline SVM & $\begin{array}{c}\text { C: } 275 \\
\text { Gamma: "scale" } \\
\text { Kernel: "rbf" }\end{array}$ \\
\hline Gaussian NB & var_smoothing: 1e-09 \\
\hline MLP & $\begin{array}{c}\text { Num_hidden_layers:2 } \\
\text { hidden_layer_sizes: [28, 28] } \\
\text { Activation: "relu" } \\
\text { max_iter: } 200 \\
\text { Solver: "adam" }\end{array}$ \\
\hline QDA & tol $=0.0001$ \\
\hline $\mathrm{RF}$ & $\begin{array}{c}\text { n_estimators:79 } \\
\text { Criterion: "entropy" }\end{array}$ \\
\hline LR & $\begin{array}{c}\text { Solver: "lbfgs" } \\
\text { C: } 1.0 \\
\text { fit_intercept: true }\end{array}$ \\
\hline
\end{tabular}

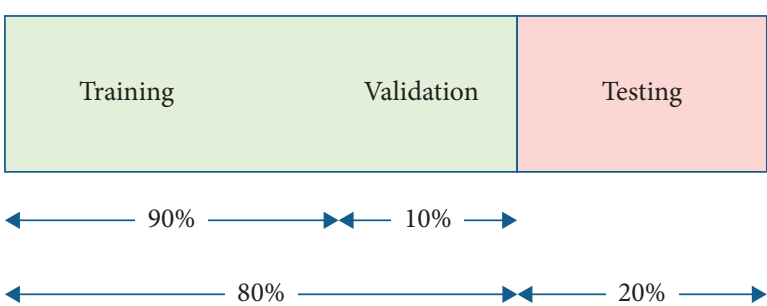

FIgURe 12: Pipeline for fragmentation of datasets.

each of the ten folds is used exactly once for validation. The optimal configurations identified in the training stage are then applied in the testing stage.

\section{Experimental Results}

Experiments are performed on an Intel Core i3 machine with $8 \mathrm{~GB}$ RAM. Python 3.9 is used as the development environment. The OpenCV 4.5 and SRGAN libraries are used for image preprocessing. MediaPipe 0.8.6 library is used as the building block for the key feature extraction. Scikit-learn 0.24.2 [39] is used for implementing the machine learning classifiers and computing the evaluation metrics for the proposed model. NumPy, Pandas, Math, OS, and Matplotlib are used as supplementary libraries. The accuracy, precision, recall, $F 1$-score, and training time are the five metrics used to evaluate the proposed framework. The training time is recorded based on the average of five runs. The proposed model is evaluated using two different datasets: $\mathrm{CK}+$ (6 classes) and JAFFE (6 classes), which are benchmark datasets for facial expression classification. For $\mathrm{CK}+, 784$ images are used for training, while 197 images are used for classification. For JAFFE, the training set contains 164 images, and the testing set contains 42 images.

To evaluate the performance of the proposed model, eight classifiers are employed to classify facial expressions across two benchmark datasets. The hyperparameters employed for each classifier are presented in Table 6. The classification is based on ten features extracted from images in each dataset using the procedure described in Section 4.

Learning curves, which determine cross-validation scores and behaviors for different training sizes for the adopted classifiers in case of $\mathrm{CK}+$, are shown in Figure 13.

The confusion matrix for each classifier on the $\mathrm{CK}+$ dataset using the proposed model is shown in Figure 14. It shows that the per-class accuracies of Anger, Happy, and Surprise classes have higher values with all classifiers than those of other emotions, while the Contempt and Sadness classes have lower per-class accuracies. Moreover, the confusion matrices for classifiers on JAFFE dataset are shown in Figure 15.

The performances of the proposed framework with eight classifiers on CK+, JAFFE, and RAF-DB [28] datasets are presented in Tables 7-9. The illustrated results show the classification report including accuracy, precision, recall, and F1-score as well as the training time taken for each classifier. A visual comparison between the classifier accuracies across the used datasets is shown in Figure 16.

Results reveal that the KNN classifier outperforms other classifiers in terms of accuracy, precision, recall, and F1- 

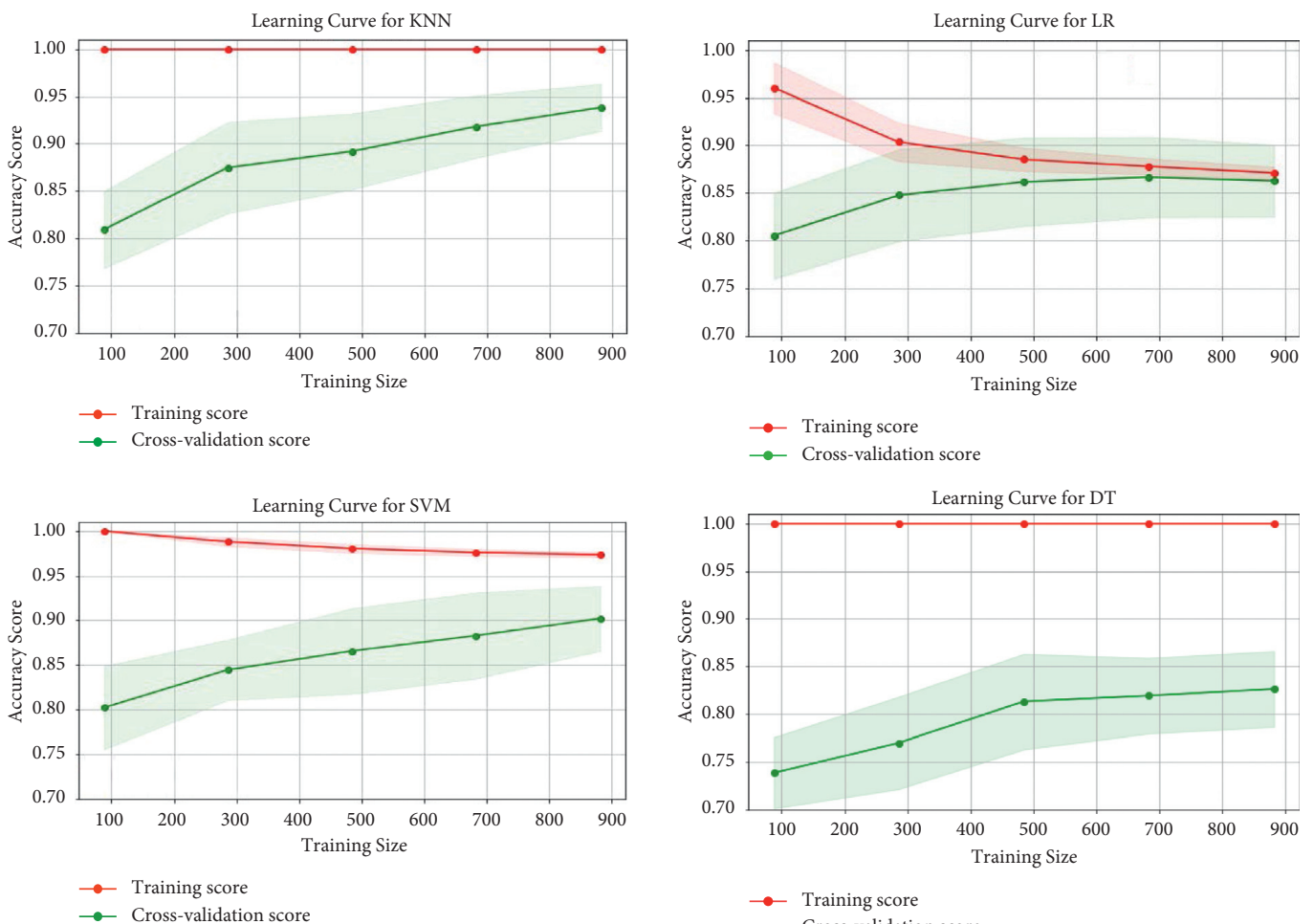

- Cross-validation score

$\rightarrow$ Training score

$\rightarrow$ Cross-validation score
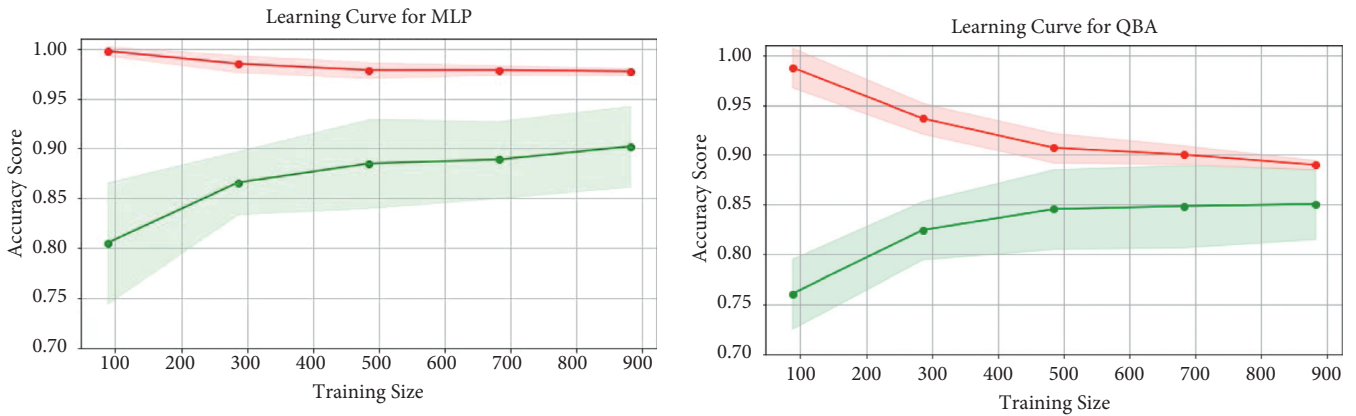

$\rightarrow$ Training score

$\rightarrow$ Cross-validation score

$\rightarrow$ Training score

$\rightarrow$ Cross-validation score
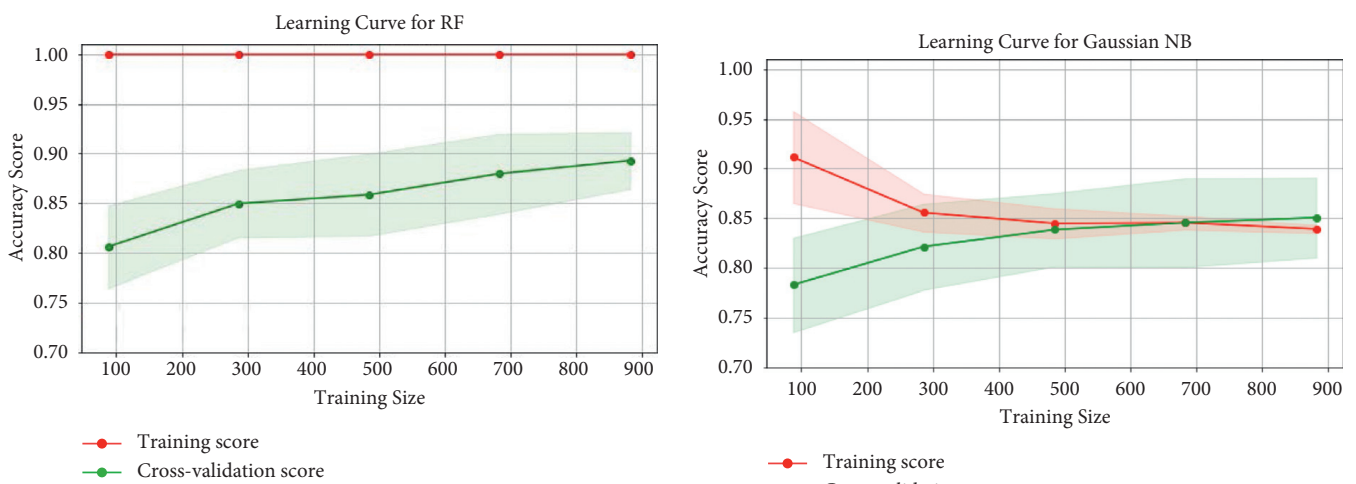

- Training score

$\rightarrow$ Cross-validation score

(a)

Figure 13: Continued. 

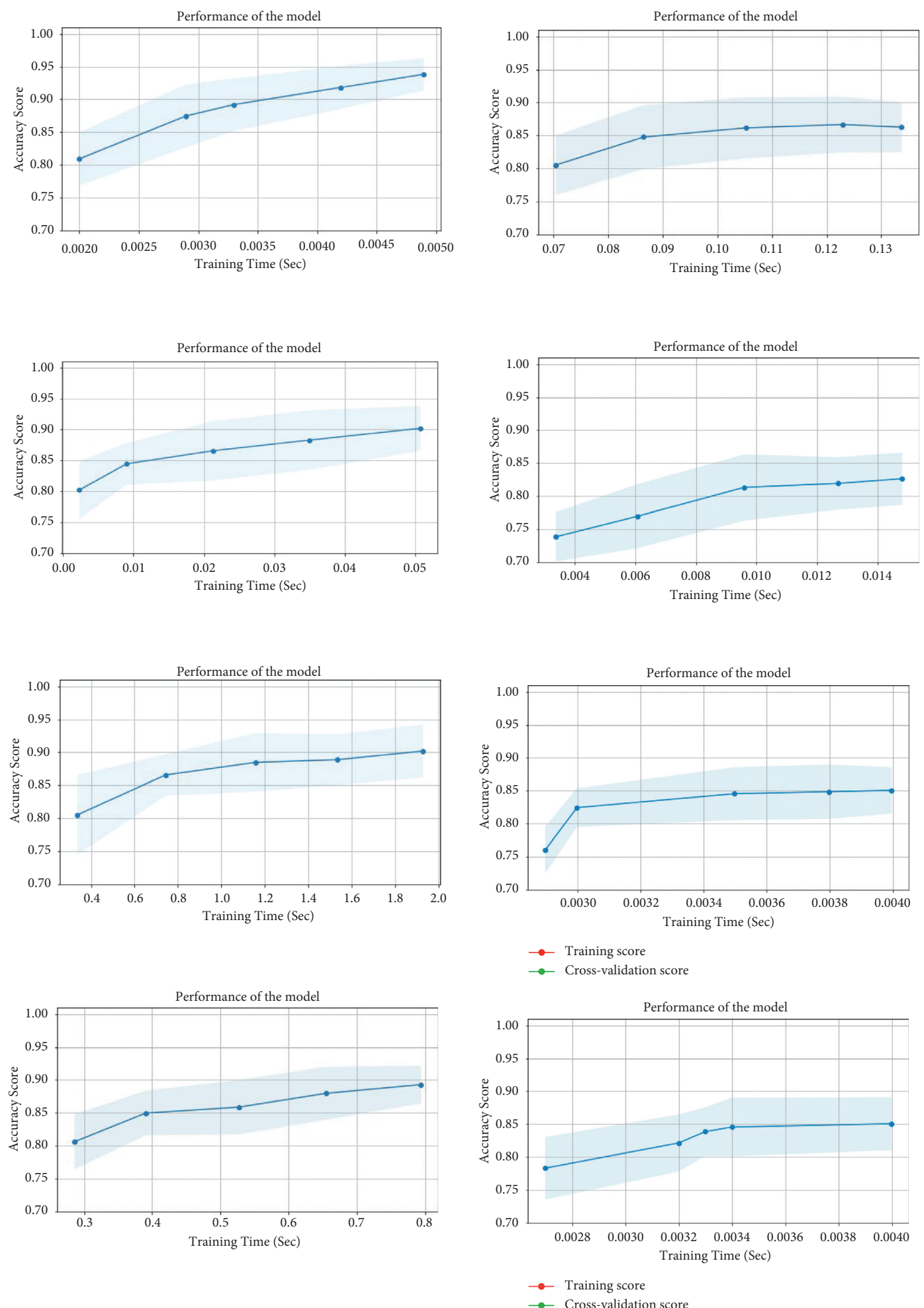

(b)

FIGURE 13: The accuracy (a) and performance vs. training time (b) for different classifiers (KNN, SVM, MLP, RF, LR, DT, QDA, and Gaussian NB) for facial expression recognition using the proposed model on $\mathrm{CK}+$ dataset. 

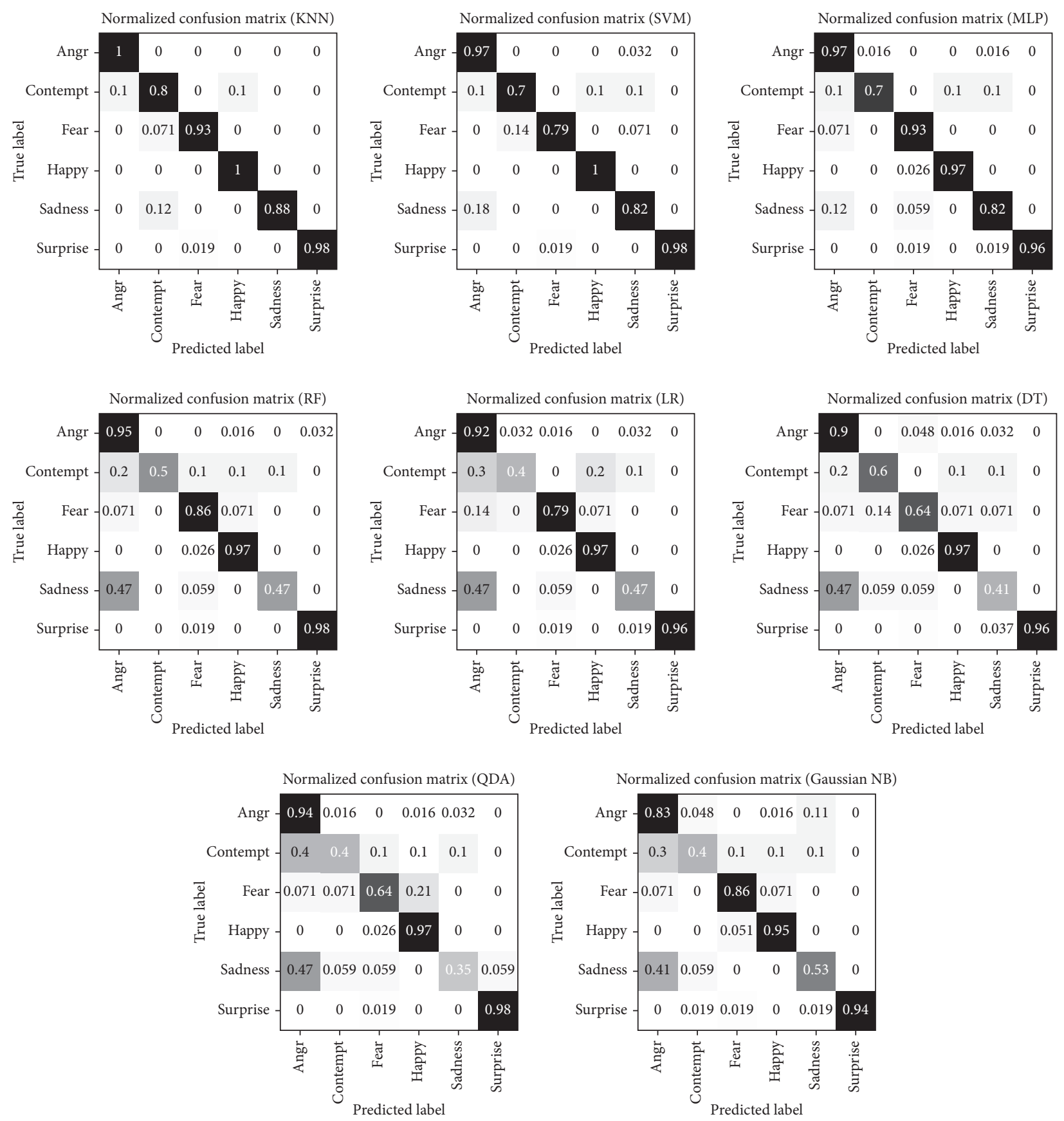

FIgure 14: Confusion matrices of emotion detection classifiers on $\mathrm{CK}+$ dataset.

score. It achieved the best accuracies of $97 \%$ and $95 \%$ on $\mathrm{CK}+$ and JAFFE datasets, respectively. The accuracies for Gaussian NB, QDA, DT, LR, RF, MLP, and SVM classifers on the $\mathrm{CK}+$ are $84 \%, 86 \%, 86 \%, 87 \%, 89 \%, 94 \%$, and $94 \%$, respectively, and those on the JAFFE are $90 \%, 79 \%, 90 \%$, $86 \%, 93 \%, 90 \%$, and $88 \%$, respectively. In addition, the time required to train the KNN and Gaussian NB is $0.005 \mathrm{sec}$ on $\mathrm{CK}+$. It is the lowest time compared to those of other classifiers. The MLP and RF classifiers have the highest training times, which are $1.82 \mathrm{sec}$ and $0.74 \mathrm{sec}$, respectively. Moreover, the proposed models are evaluated on theRAFDB. The results of this evaluation reveal that the proposed MLP and SVM models can be considered as good emotion detection models for this database, especially with an accuracy of $67 \%$ for both models. Therefore, the proposed approach provides a variety of models, which are optimal for robust emotion detection environments.

\section{Discussion}

The simulation results reveal that the proposed approach shows a high performance in human emotion detection. Furthermore, they clarify that the proposed encoding module has a superior performance with the deployed classifiers including KNN, SVM, and MLP. In this section, a brief comparison is presented between the proposed 


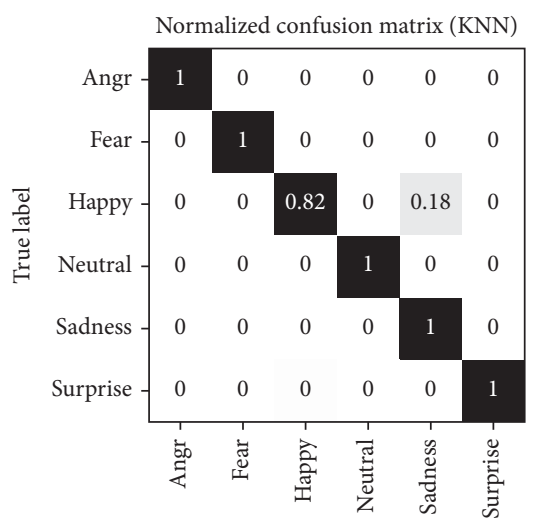

Predicted label

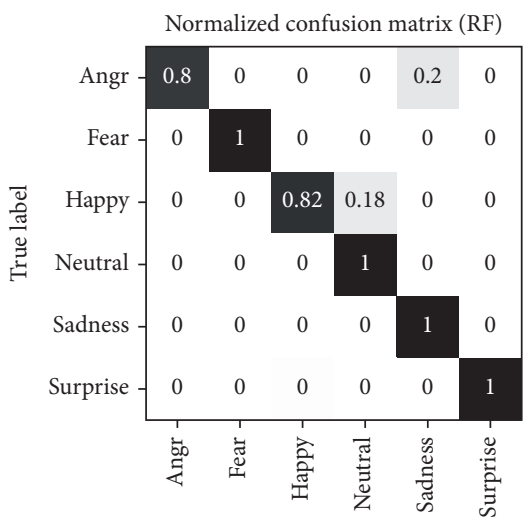

Predicted label

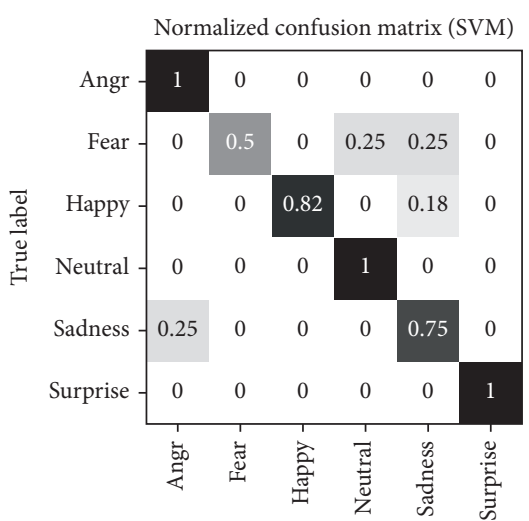

Predicted label

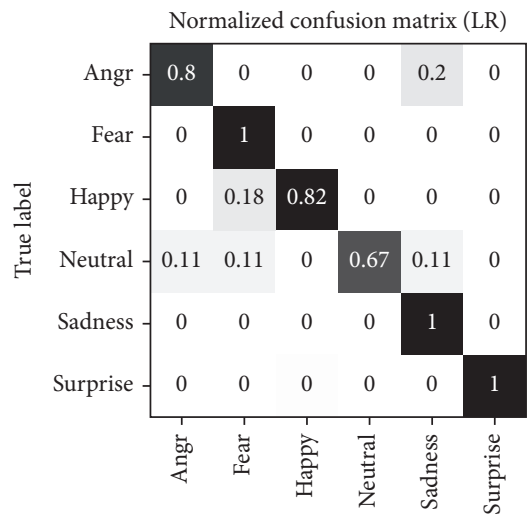

Predicted label

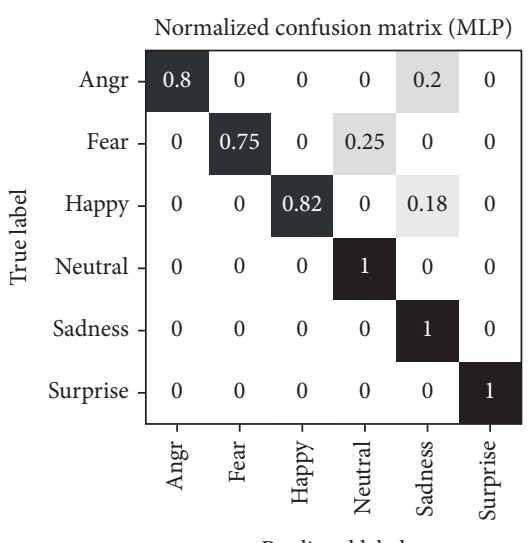

Predicted label

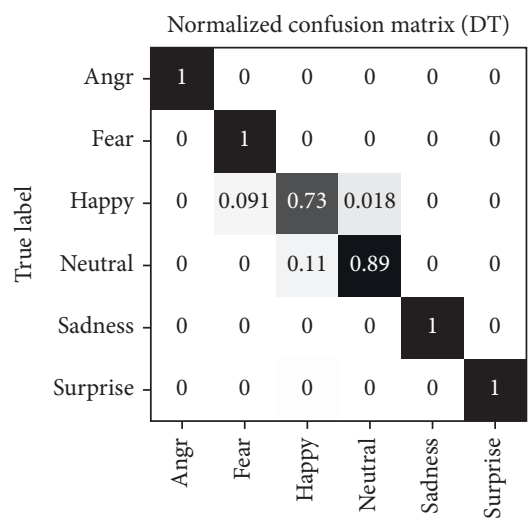

Predicted label

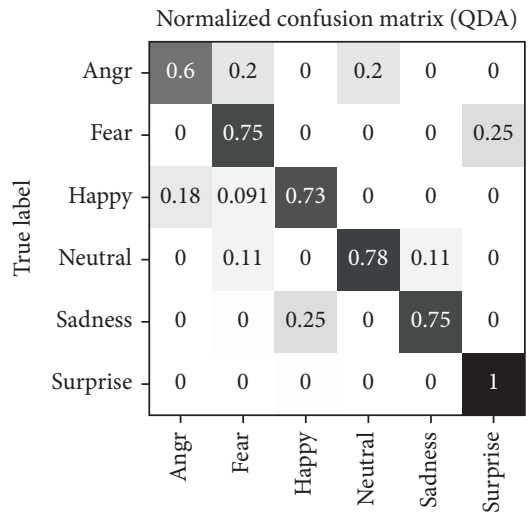

Predicted label

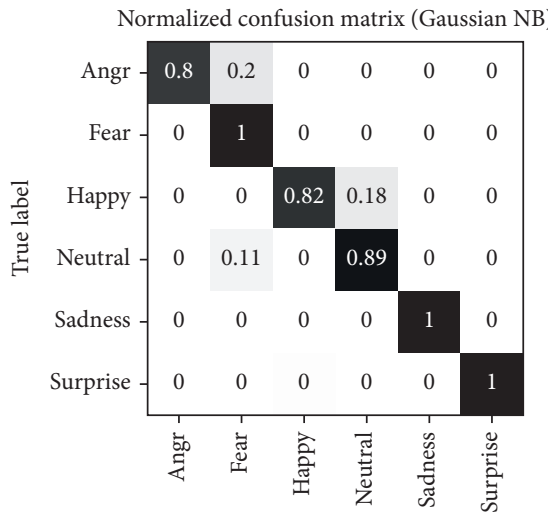

Predicted label

FIGURE 15: Confusion matrices of emotion detection classifiers on JAFFE dataset.

TABle 7: Comparison of eight ML classifiers on CK+ dataset.

\begin{tabular}{lccccc}
\hline Classifier & Classification accuracy & Precision & Recall & F1-score & Training time (seconds) \\
\hline Gaussian NB & 0.84 & 0.84 & 0.84 & 0.84 & 0.005 \\
QDA & 0.86 & 0.85 & 0.86 & 0.85 & 0.006 \\
DT & 0.86 & 0.85 & 0.86 & 0.85 & 0.018 \\
LR & 0.87 & 0.86 & 0.87 & 0.86 & 0.15 \\
RF & 0.89 & 0.90 & 0.89 & 0.88 & 0.74 \\
MLP & 0.94 & 0.94 & 0.94 & 0.94 & 1.82 \\
SVM & 0.94 & 0.94 & 0.94 & 0.94 & 0.06 \\
KNN & 0.97 & 0.97 & 0.97 & 0.97 & 0.005 \\
\hline
\end{tabular}


TABle 8: Comparison of eight ML classifiers on JAFFE dataset.

\begin{tabular}{lccccc}
\hline Classifier & Classification accuracy & Precision & Recall & F1-score & Training time (seconds) \\
\hline Gaussian NB & 0.90 & 0.93 & 0.90 & 0.91 & 0.005 \\
QDA & 0.79 & 0.80 & 0.79 & 0.79 & 0.005 \\
DT & 0.90 & 0.91 & 0.90 & 0.90 & 0.005 \\
LR & 0.86 & 0.90 & 0.86 & 0.86 & 0.09 \\
RF & 0.93 & 0.94 & 0.93 & 0.93 & 0.33 \\
MLP & 0.90 & 0.94 & 0.90 & 0.91 & 0.41 \\
SVM & 0.88 & 0.91 & 0.88 & 0.88 & 0.008 \\
KNN & 0.95 & 0.97 & 0.95 & 0.95 & 0.002 \\
\hline
\end{tabular}

TABLE 9: Comparison of eight ML classifiers on RAF-DB.

\begin{tabular}{lccccc}
\hline Classifier & Classification accuracy & Precision & Recall & F1-score & Training time (seconds) \\
\hline Gaussian NB & 0.65 & 0.62 & 0.65 & 0.61 & 0.01 \\
QDA & 0.64 & 0.62 & 0.64 & 0.61 & 0.02 \\
DT & 0.53 & 0.52 & 0.53 & 0.52 & 0.26 \\
LR & 0.66 & 0.62 & 0.66 & 0.63 & 0.9 \\
RF & 0.65 & 0.61 & 0.65 & 0.62 & 11 \\
MLP & 0.67 & 0.64 & 0.67 & 0.64 & 20 \\
SVM & 0.67 & 0.64 & 0.67 & 0.64 & 0.053 \\
KNN & 0.63 & 0.60 & 0.63 & 0.61 & 0.053 \\
\hline
\end{tabular}

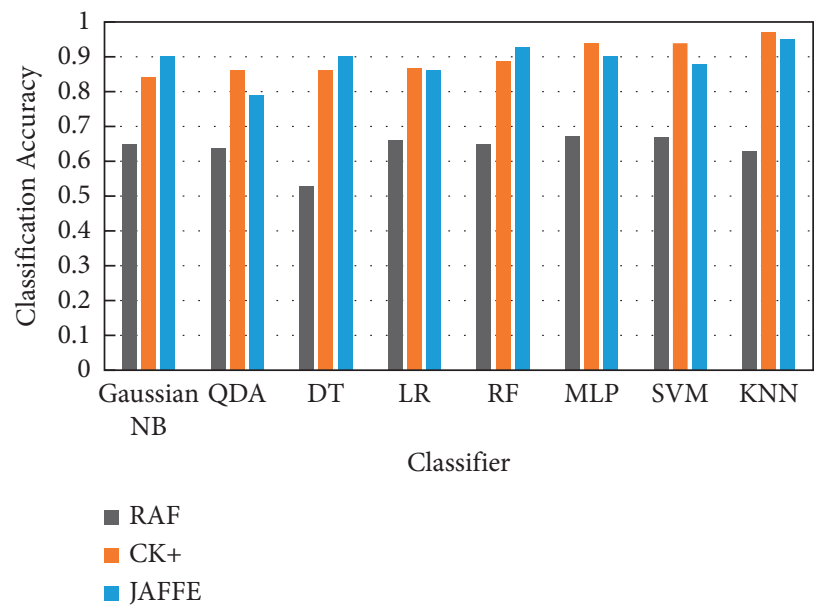

Figure 16: Accuracies of eight ML classifiers on RAF, CK+, and JAFFE datasets for facial expression classification using the proposed approach.

TABLE 10: Comparison between the proposed work and the state-of-the-art works.

\begin{tabular}{|c|c|c|c|c|c|c|}
\hline \multirow{2}{*}{ Work } & \multirow{2}{*}{ Year } & \multirow{2}{*}{ Method } & \multicolumn{3}{|c|}{ Accuracy (\%) } & \multirow{2}{*}{ Time (s) } \\
\hline & & & JAFFE & $\mathrm{CK}+$ & RAF & \\
\hline [40] & 2018 & CNN & 50.12 & 93.64 & & - \\
\hline \multirow{2}{*}[28]{} & \multirow{2}{*}{2019} & $\mathrm{mSVM}$ & 88.95 & 91.98 & 65.12 & - \\
\hline & & LDA & 83.45 & 92.33 & 56.93 & - \\
\hline$[41]$ & 2018 & $\mathrm{RF}$ & - & 93.4 & - & - \\
\hline$[42]$ & 2018 & SVM & - & 95.8 & - & - \\
\hline \multirow{2}{*}[43]{} & \multirow{2}{*}{2018} & AlexNet & 93 & 90.2 & - & - \\
\hline & & VGG16 & 96 & 92.4 & - & 0.94 \\
\hline$[44]$ & 2018 & VGG19 & 93 & 93 & - & - \\
\hline$[45]$ & 2021 & $\mathrm{CNN}$ & 96.8 & 86.5 & - & 62.5 \\
\hline \multirow{2}{*}{ [46] } & \multirow{2}{*}{2018} & AlexNet & - & - & 55.6 & - \\
\hline & & VGG & - & - & 58.2 & - \\
\hline \multirow{4}{*}{ Proposed } & \multirow{4}{*}{2021} & MLP & 90 & 94 & 67 & 1.12 \\
\hline & & SVM & 88 & 94 & 67 & 0.034 \\
\hline & & $\mathrm{KNN}$ & 95 & 97 & 63 & 0.004 \\
\hline & & LR & 86 & 87 & 66 & 0.12 \\
\hline
\end{tabular}


approach and the works in the literature as illustrated in Table 10. It can be observed that the proposed approach has a superior performance among the efforts in this field.

\section{Conclusion}

The issue of Human-Robot Interaction (HRI) has been discussed in this paper. As a solution, the paper presented a novel approach for facial expression recognition. This proposed approach consists of four phases, which are carried out to extract key points from facial images using a real-time algorithm (MediaPipe). Furthermore, these key points are enrolled into a sequence of selection, mesh generator, and angular encoding modules. Moreover, the generated feature maps are classified using several classification algorithms, including SVM, KNN, RF, QDA, NB, LR, DT, and MLP. The novelty of the proposed approach is highlighted in the proposed key point analysis and angular encoding algorithm. This algorithm is efficient, because it generates only ten features (angular values), which are discriminative for different emotional classification categories. The proposed approach has been evaluated on CK+, JAFEE, and RAF-DB datasets. It reveals a superior performance in terms of accuracy of detection and processing time evaluation metrics. Furthermore, the low dimensionality of extracted features enables the ML-based approaches to reach an optimum performance in a short time with much lower computational cost than those of the DL-based approaches, which require more time for convergence and need much computational cost.

In addition, the future work that can be deduced from this paper is introducing a method for emotion detection from other modalities such as videos, spoken words, and written text. Furthermore, hardware implementation of the proposed approach is a research trend, which we are working on. Moreover, further machine learning techniques such as dictionary learning and semi-supervised learning can be performed to solve this issue.

\section{Data Availability}

The data used to support the findings of this study are available from the corresponding author upon request.

\section{Conflicts of Interest}

The authors declare that there are no conflicts of interest regarding the publication of this paper.

\section{Acknowledgments}

This research was funded by the Deanship of Scientific Research at Princess Nourah bint Abdulrahman University through the Fast-Track Research Funding Program to support publication in the top Journal (Grant no. 42-FTTJ38).

\section{References}

[1] C.-H. Chen, I.-J. Lee, and L.-Y. Lin, "Augmented reality-based self-facial modeling to promote the emotional expression and social skills of adolescents with autism spectrum disorders," Research in Developmental Disabilities, vol. 36, pp. 396-403, 2015.

[2] S. Hickson, N. Dufour, A. Sud, V. Kwatra, and I. Essa, "Eyemotion: classifying facial expressions in VR using eyetracking cameras," in Proceedings of the 2019 IEEE Winter Conference on Applications of Computer Vision (WACV), pp. 1626-1635, Waikoloa, HI, USA, January 2019.

[3] M. A. Assari and M. Rahmati, "Driver drowsiness detection using face expression recognition," in Proceedings of the 2011 IEEE International Conference on Signal and Image Processing Applications (ICSIPA), pp. 337-341, Kuala Lumpur, Malaysia, November 2011.

[4] T. B. Sheridan, "Human-robot interaction," Human Factors: The Journal of the Human Factors and Ergonomics Society, vol. 58, no. 4, pp. 525-532, 2016.

[5] X. Zhu, X. Li, and S. Zhang, "Block-row sparse multiview multilabel learning for image classification," IEEE Transactions on Cybernetics, vol. 46, no. 2, pp. 450-461, 2015.

[6] T. Chen and K.-H. Yap, "Discriminative BoW framework for mobile landmark recognition," IEEE Transactions on Cybernetics, vol. 44, no. 5, pp. 695-706, 2013.

[7] T. Bai, Y.-F. Li, and X. Zhou, "Learning local appearances with sparse representation for robust and fast visual tracking," IEEE Transactions on Cybernetics, vol. 45, no. 4, pp. 663-675, 2014.

[8] K. Han, D. Yu, and I. Tashev, "Speech emotion recognition using deep neural network and extreme learning machine," in Proceedings of the Fifteenth Annual Conference of the International Speech Communication Association, Singapore, September 2014.

[9] T. Abegaz, G. Dozier, K. Bryant et al., "Hybrid GAs for Eigenbased facial recognition," in Proceedings of the 2011 IEEE Workshop on Computational Intelligence in Biometrics and Identity Management (CIBIM), pp. 127-130, Paris, France, April 2011.

[10] W. Y. Zhao and R. Chellappa, "SFS based view synthesis for robust face recognition," in Proceedings of the Fourth IEEE International Conference on Automatic Face and Gesture Recognition, pp. 285-292, Grenoble, France, March 2000.

[11] B. Gökberk, A. A. Salah, and L. Akarun, "Rank-based decision fusion for 3D shape-based face recognition," in Proceedings of the International Conference on Audio-and Video-Based Biometric Person Authentication, pp. 1019-1028, Hilton Rye Town, NY, USA, July 2005.

[12] V. I. Pavlovic, R. Sharma, and T. S. Huang, "Visual interpretation of hand gestures for human-computer interaction: a review," IEEE Transactions on Pattern Analysis and Machine Intelligence, vol. 19, no. 7, pp. 677-695, 1997.

[13] S. Ghosh, A. Dhall, and N. Sebe, "Automatic group affect analysis in images via visual attribute and feature networks," in Proceedings of the 2018 25th IEEE International Conference on Image Processing (ICIP), pp. 1967-1971, Athens, Greece, October 2018.

[14] A. Mehrabian, "Communication without words," in Communication Theory, pp. 193-200, Routledge, UK, London, 2017.

[15] A. Glowacz, "Ventilation diagnosis of angle grinder using thermal imaging," Sensors, vol. 21, no. 8, Article ID 2853, 2021.

[16] B. Abd El-Rahiem, A. Sedik, G. M. El Banby et al., "An efficient deep learning model for classification of thermal face 
images," Journal of Enterprise Information Management, vol. ahead-of-print, no. ahead-of-print, 2020.

[17] A. Glowacz, "Fault diagnosis of electric impact drills using thermal imaging," Measurement, vol. 171, Article ID 108815, 2021.

[18] A. I. Siam, N. A. El-Bahnasawy, G. M. El Banby, A. Abou Elazm, and F. E. Abd El-Samie, "Efficient video-based breathing pattern and respiration rate monitoring for remote health monitoring," Journal of the Optical Society of America A, vol. 37, no. 11, Article ID C118, 2020.

[19] J. Deng, G. Pang, Z. Zhang, Z. Pang, H. Yang, and G. Yang, "cGAN based facial expression recognition for human-robot interaction," IEEE Access, vol. 7, pp. 9848-9859, 2019.

[20] D. V. Sang, L. T. B. Cuong, and V. Van Thieu, "Multi-task learning for smile detection, emotion recognition and gender classification," in Proceedings of the Eighth International Symposium on Information and Communication Technology, pp. 340-347, New York, NY, USA, December 2017.

[21] K. Shan, J. Guo, W. You, D. Lu, and R. Bie, "Automatic facial expression recognition based on a deep convolutional-neuralnetwork structure," in Proceedings of the 2017 IEEE 15th International Conference on Software Engineering Research, Management and Applications (SERA), pp. 123-128, London, UK, June 2017.

[22] E. O. Ige, K. Debattista, and A. Chalmers, "Towards HDR based facial expression recognition under complex lighting," in Proceedings of the 33rd Computer Graphics International, pp. 49-52, Poole, UK, September 2016.

[23] S. Berretti, B. Ben Amor, M. Daoudi, and A. Del Bimbo, “3D facial expression recognition using SIFT descriptors of automatically detected keypoints," The Visual Computer, vol. 27, no. 11, pp. 1021-1036, 2011.

[24] S. Zhang, X. Pan, Y. Cui, X. Zhao, and L. Liu, "Learning affective video features for facial expression recognition via hybrid deep learning," IEEE Access, vol. 7, pp. 32297-32304, 2019.

[25] X. Pan, G. Ying, G. Chen, H. Li, and W. Li, “A deep spatial and temporal aggregation framework for video-based facial expression recognition," IEEE Access, vol. 7, pp. 48807-48815, 2019.

[26] P. Lucey, J. F. Cohn, T. Kanade, J. Saragih, Z. Ambadar, and I. Matthews, "The extended cohn-kanade dataset (CK+): a complete dataset for action unit and emotion-specified expression," in Proceedings of the 2010 IEEE Computer Society Conference on Computer Vision and Pattern RecognitionWorkshops, pp. 94-101, San Francisco, CA, USA, June 2010.

[27] M. Lyons, S. Akamatsu, M. Kamachi, and J. Gyoba, "Coding facial expressions with Gabor wavelets," in Proceedings of the Third IEEE International Conference on Automatic Face and Gesture Recognition, pp. 200-205, Nara, Japan, April 1998.

[28] S. Li and W. Deng, "Reliable crowdsourcing and deep localitypreserving learning for unconstrained facial expression recognition," IEEE Transactions on Image Processing, vol. 28, no. 1, pp. 356-370, 2019.

[29] C. Ledig, L. Theis, F. Huszar et al., "Photo-realistic single image super-resolution using a generative adversarial network," in Proceedings of the IEEE conference on computer vision and pattern recognition, pp. 4681-4690, Honolulu, HI, USA, July 2017.

[30] Mediapipe, 2021, https://google.github.io/mediapipe/.

[31] C. Lugaresi, J. Tang, H. Nash et al., "Mediapipe: a framework for building perception pipelines," 2019, https://arxiv.org/abs/ 1906.08172 .
[32] Y. Kartynnik, A. Ablavatski, I. Grishchenko, and M. Grundmann, "Real-time facial surface geometry from monocular video on mobile GPUs," 2019, https://arxiv.org/ abs/1907.06724.

[33] P. Ekman, Facial Action Coding System, Consulting Psychologists Palo Alto, Palo Alto, CA, USA, 1978.

[34] P. Ekman and W. V Friesen, Manual of the Facial Action Coding System (FACS), Consulting Psychologists Press, Palo Alto, CA, USA, Trans edition, 1978.

[35] J. Hamm, C. G. Kohler, R. C. Gur, and R. Verma, “Automated Facial Action Coding System for dynamic analysis of facial expressions in neuropsychiatric disorders," Journal of Neuroscience Methods, vol. 200, no. 2, pp. 237-256, 2011.

[36] FACS-cheat sheet, 2021, https://melindaozel.com/facs-cheatsheet/.

[37] K. Crammer and Y. Singer, "On the algorithmic implementation of multiclass kernel-based vector machines," Journal of Machine Learning Research, vol. 2, pp. 265-292, 2001.

[38] L. Li, K. Jamieson, G. DeSalvo, A. Rostamizadeh, and A. Talwalkar, "Hyperband: a novel bandit-based approach to hyperparameter optimization," Journal of Machine Learning Research, vol. 18, no. 1, pp. 6765-6816, 2017.

[39] F. Pedregosa, G. Varoquaux, A. Gramfort et al., "Scikit-learn: machine learning in Python," The Journal of Machine Learning Research, vol. 12, pp. 2825-2830, 2011.

[40] P. M. Ferreira, F. Marques, J. S. Cardoso, and A. Rebelo, "Physiological inspired deep neural networks for emotion recognition," IEEE Access, vol. 6, pp. 53930-53943, 2018.

[41] A. Dapogny, K. Bailly, and S. Dubuisson, "Confidenceweighted local expression predictions for occlusion handling in expression recognition and action unit detection," International Journal of Computer Vision, vol. 126, no. 2, pp. 255-271, 2018.

[42] S. Agarwal, B. Santra, and D. P. Mukherjee, "Anubhav: recognizing emotions through facial expression," The Visual Computer, vol. 34, no. 2, pp. 177-191, 2018.

[43] B. Verma and A. Choudhary, "A framework for driver emotion recognition using deep learning and Grassmann manifolds," in Proceedings of the 2018 21st International Conference on Intelligent Transportation Systems (ITSC), pp. 1421-1426, Maui, HI, USA, November 2018.

[44] S. Ramalingam and F. Garzia, "Facial expression recognition using transfer learning," in Proceedings of the 2018 International Carnahan Conference on Security Technology (ICCST), pp. 1-5, Montreal, QC, Canada, October 2018.

[45] Y. K. Bhatti, A. Jamil, N. Nida, M. H. Yousaf, S. Viriri, and S. A. Velastin, "Facial expression recognition of instructor using deep features and extreme learning machine," Computational Intelligence and Neuroscience, vol. 2021, Article ID 5570870, 17 pages, 2021.

[46] S. Zhao, H. Cai, H. Liu, J. Zhang, and S. Chen, "Feature selection mechanism in $\mathrm{CNNs}$ for facial expression recognition," in Proceedings of the British Machine Vision Conference, Newcastle, UK, September 2018. 